
\title{
Numerical Study on FRP Wrapped Concrete Columns Under Compression
}

\author{
P. Sangeetha' and M. Shanmugapriya ${ }^{2}$ \\ 'Department of Civil Engineering, Sri Sivasubramaniya Nadar College of Engineering, Kalavakkam - 603110, \\ Tamil Nadu, India; sangeethap@ssn.edu.in \\ ${ }^{2}$ Department of Mathematics, Sri Sivasubramaniya Nadar College of Engineering, Kalavakkam - 603110, \\ Tamil Nadu, India; shanmugapriyam@ssn.edu.in
}

\begin{abstract}
Fiber Reinforced Plastic (FRP) wrapped concrete column shows better performance in terms of strength and ductility of the columns. Objective: Already published experimental results of the FRP wrapped concrete columns were used to analyse the same using Finite Element Software ANSYS and ANN. The comparison between the ANSYS and ANN results were made. Methods/Analysis: The cross sections of the concrete column varied in the study were square, circular and rectangular with different corner radius. The software stimulates the behaviour of the FRP wrapped concrete column properly if proper choice of elements from the ANSYS library were made. Findings: Results from the analysis of the specimens made from the quarter part of the model showed that confinement of the FRP over the concrete columns increases the strength and ductility by $25 \%$ when compared to unconfined columns. Applications: Anartificial Neural Networks (ANNs) approach was also used to find the compressive stress of the confined and unconfined columns by using both experimental and analytical results.
\end{abstract}

Keywords: Compressive Strength, FRP Columns, Plies, ANSYS, ANN

\section{Introduction}

The retro fitting of the concrete columns has been used as technique to improve the structural and ductility behaviour of the concrete columns. In Japan, researcher reported that fiber wrapped concrete columns have been successful by using many fibers like glass, aramid fibers and carbon fibers over the structures like piers, columns, chimneys etc ${ }^{1}$. Many researchers ${ }^{2-8}$ have proposed several models to strengthen the square and circular concrete columns with FRP fibers.

$\mathrm{In}^{9}$ have developed quarter finite element model of the confined and un confined columns to investigate the behaviour of the concrete columns wrapped with number of plies of carbon and aramid fibers.Sangeetha ${ }^{10-13}$ have analysed the FRP wrapped concrete columns and composite againstpublished experimental results obtained using finite element software ANSYS. In the recent years, Artificial Neural Network (ANN) has been used to predict the behaviour of various civil Engineering structures. The regression analysis using $\mathrm{ANN}$ approach is able to predict the stress developed in the FRP wrapped concrete columns under compression. This paper gives the comparison made between the predicted compressive strength of the confined and unconfined concrete columns using ANN approach with published experimental and analytical behaviour of the specimens with varying cross section, number of plies and corner radius of the column.

\section{Analytical Model}

Column model were made of plain cement concrete having compressive strength of M35, M40 \&M45. The parameters varied in the study are cross sectional shape

*Author for correspondence 
of the column (square-S, rectangle - $\mathrm{R}$ and circle - C), Types of fibers (Carbon-C and Aramid -A), Number of plies (0 - unconfined, 2, 3, 4, 5, 6, $9 \& 12$ plies confined), Compressive strength of concrete $(35,40 \& 45 \mathrm{Mpa})$ and corner radius $(5,25,38,100 \& 150 \mathrm{~mm})$. Totally sixty five specimens were analysed using ANSYS. Only quarter model were used for analysis because of symmetry in order to reduce the memory required for analysis. SOLID 186 is used for modelling concrete portion and SHELL93 element is used for fibers. Bottom end of the columns was fixed and top surfacewas subjected to axial compressive load. Figure 1 shows the quarter mesh model of the specimen. Figure 2 shows the stress distribution along Y- direction of the square (S38-C3), rectangular (R25C3) and circular column (C150-A12) obtained from the analysis using ANSYS.
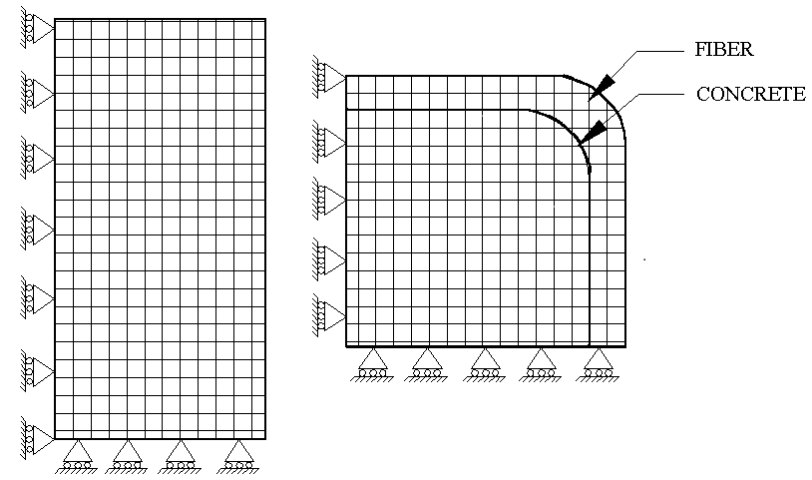

Figure 1. Meshed quarter model of the FRP wrapped column.

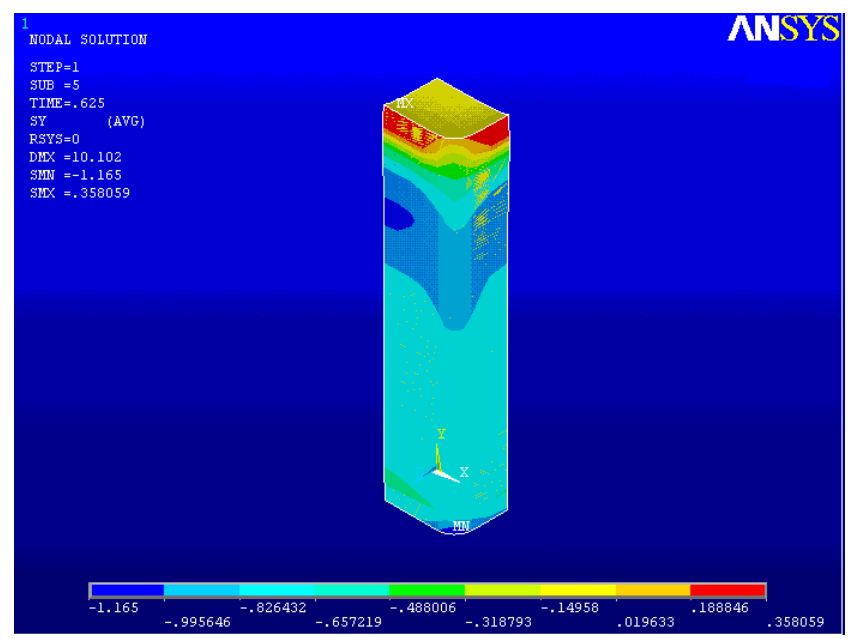

Square Column-S38-C3

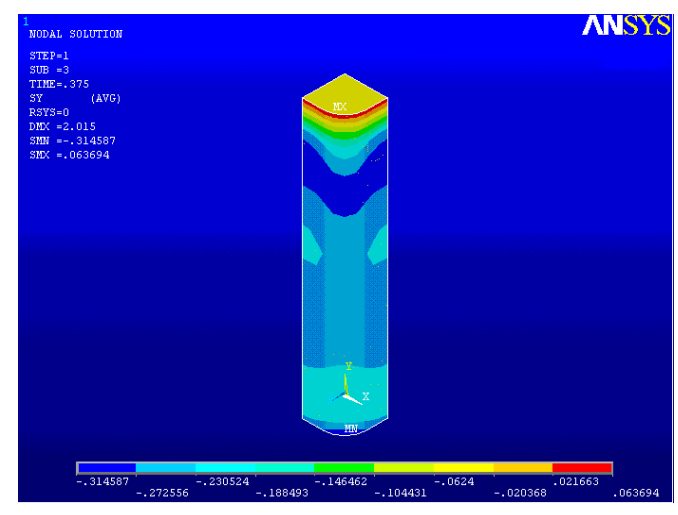

Rectangular Column - R25-C3

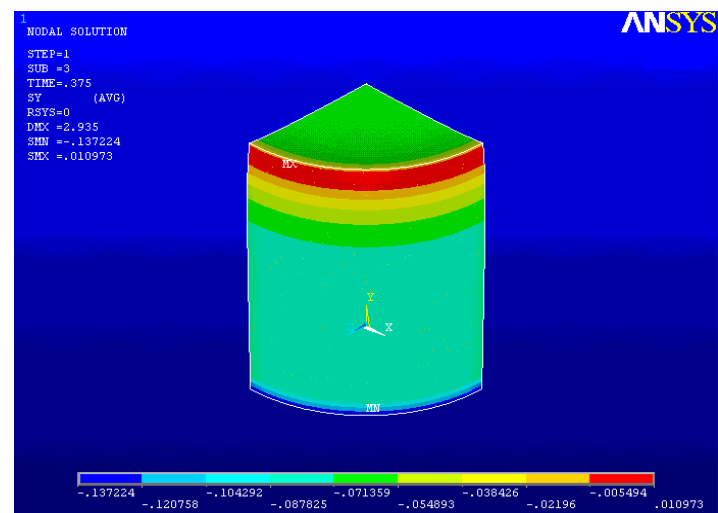

Circular Column -C150-A12

Figure 2. Stress along Y- direction of FRP wrapped concrete column.

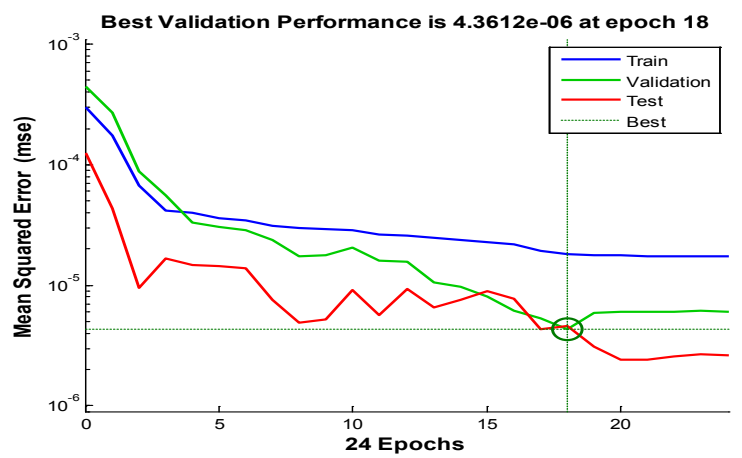

Figure 3. Performance of the Square and Rectangular columns confined with FRP sheets.

\section{Result and Discussion}

The results obtained from training the specimens using ANN are showed in Figure 3 to 7. Figure 3 and Figure 4 shows the error of the network starting from larger 
value and getting decrease for the lower value in terms of mean square. From the Figure 4 it is observed that network being learned. The three coloured lines in the plot are because of the sixty four specimen results as input and targets were accordingly divided into three different sets. The training is continuous upto the training vector reduces the error and finally reaches the validation vectors and then the training is been stopped. Figure 5 and 6 shows the training of the specimens. Figure 7 shows the error stimulated by ANN for the specimens. Table 1 gives the comparison of the results between experimental, analytical and ANN results of the all specimens.

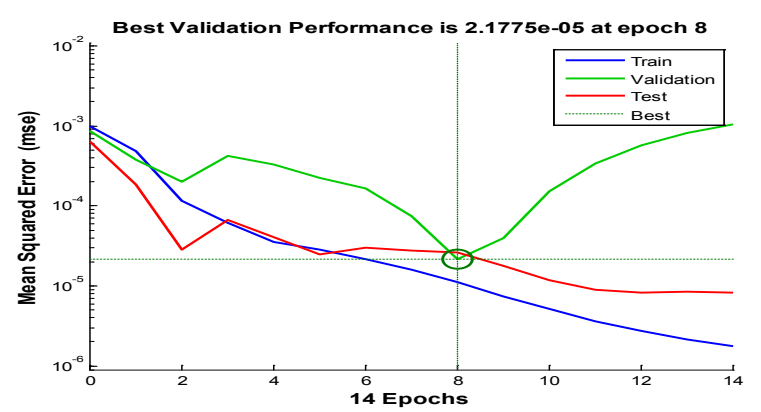

Figure 4. Performance of the circular columns confined with FRP sheets.
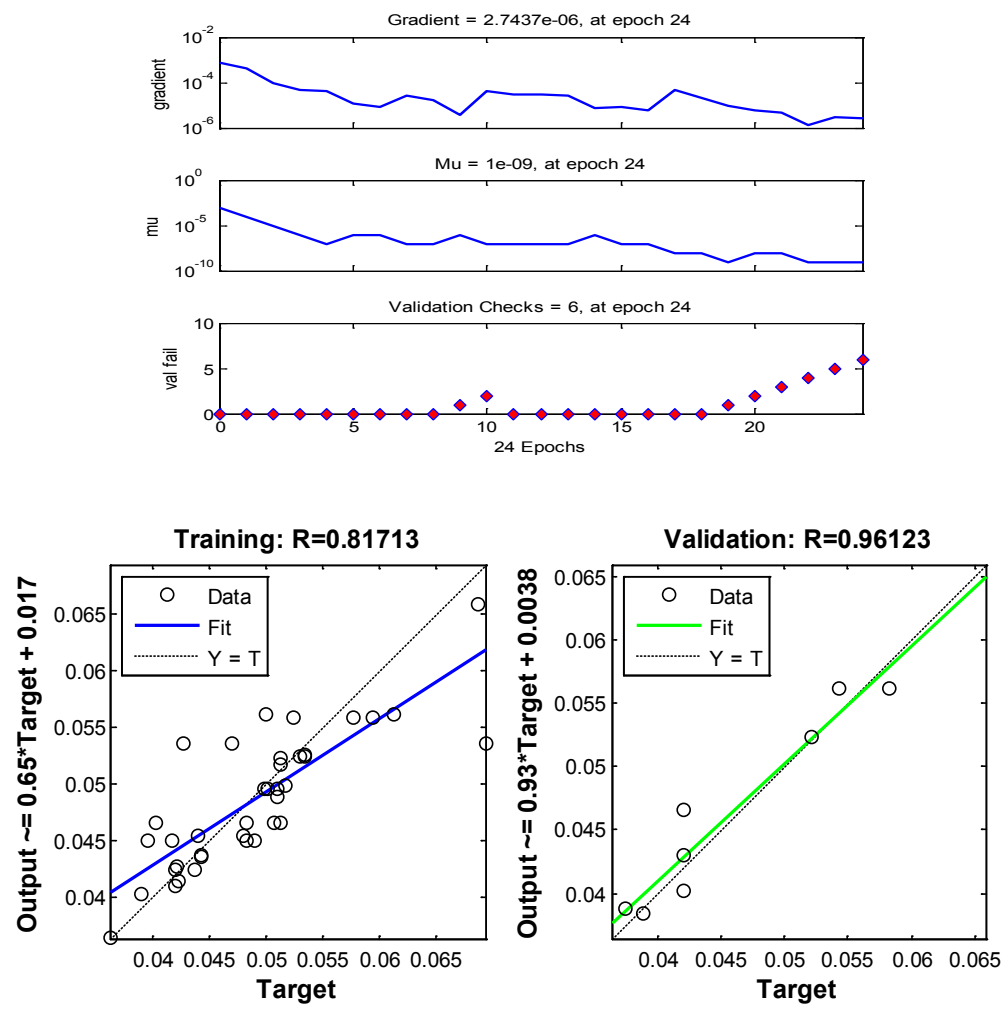

Test: $\mathbf{R}=0.95489$
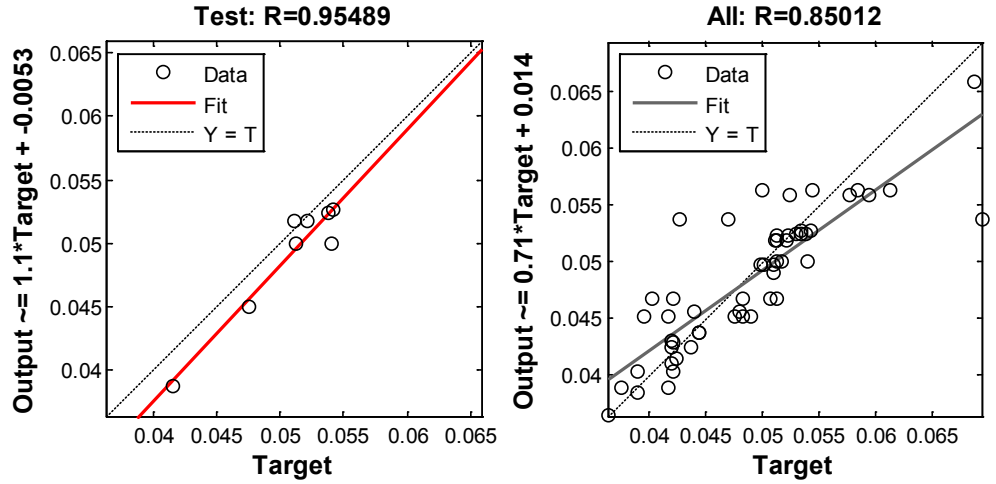

Figure 5. Regressions of training, validation and test data for square and rectangular shaped column. 
Table 1. Comparison between experimental, analytical and ANN results and its error

\begin{tabular}{|c|c|c|c|c|c|c|c|c|}
\hline \multirow[t]{2}{*}{ Sl.No } & \multirow[t]{2}{*}{ Model } & Geometry & \multirow[t]{2}{*}{$\begin{array}{l}\text { No. of } \\
\text { Plies }\end{array}$} & $\begin{array}{l}\text { Concrete } \\
\text { strength }\end{array}$ & $\begin{array}{l}\text { Experimental } \\
\text { Axial Stress }\end{array}$ & $\begin{array}{l}\text { Analytical } \\
\text { Axial Stress }\end{array}$ & ANN & \multirow[t]{2}{*}{ Error } \\
\hline & & $\begin{array}{l}1 \times b \times h \\
(\mathrm{~mm})\end{array}$ & & (Mpa) & \multicolumn{3}{|l|}{$\left(\mathrm{kN} / \mathbf{m m}^{2}\right)$} & \\
\hline 1 & S - NC- 40 & $152^{\prime} 152 \times 500$ & 0 & 40 & 0.0416 & 0.0401 & 0.038789 & 0.002811 \\
\hline 2 & S - NC - 45 & $152^{\prime} 152 \times 500$ & 0 & 45 & 0.0423 & 0.0412 & 0.041391 & 0.000909 \\
\hline 3 & S5-C3 - 40 & $152^{\prime} 152 \times 500$ & 3 & 40 & 0.0395 & 0.0421 & 0.045052 & -0.00555 \\
\hline 4 & S5-C4 - 45 & $152^{\prime} 152 \times 500$ & 3 & 45 & 0.0402 & 0.0416 & 0.046621 & -0.00642 \\
\hline 5 & S25-C3 - 40 & $152^{\prime} 152 \times 500$ & 3 & 40 & 0.0416 & 0.0450 & 0.045052 & -0.00345 \\
\hline 6 & S25-C3 - 45 & $152^{\prime} 152 \times 500$ & 3 & 45 & 0.0421 & 0.0470 & 0.046621 & -0.00452 \\
\hline 7 & S38-C3 - 40 & $152^{\prime} 152 \times 500$ & 3 & 40 & 0.0475 & 0.0450 & 0.045052 & 0.002448 \\
\hline 8 & S38-C3 - 45 & $152^{\prime} 152 \times 500$ & 3 & 45 & 0.0482 & 0.0475 & 0.046621 & 0.001579 \\
\hline 9 & S5-C5 - 40 & $152^{\prime} 152 \times 500$ & 5 & 40 & 0.0427 & 0.0431 & 0.053632 & -0.01093 \\
\hline 10 & S5-C5 - 45 & $152^{\prime} 152 \times 500$ & 5 & 45 & 0.0439 & 0.0461 & 0.045509 & -0.00161 \\
\hline 11 & S25-C4- 40 & $152^{\prime} 152 \times 500$ & 4 & 40 & 0.05 & 0.0510 & 0.056222 & -0.00622 \\
\hline 12 & S25-C4- 45 & $152^{\prime} 152 \times 500$ & 4 & 45 & 0.0509 & 0.0473 & 0.04894 & 0.00196 \\
\hline 13 & S25-C5 - 40 & $152^{\prime} 152 \times 500$ & 5 & 40 & 0.0469 & 0.0417 & 0.053632 & -0.00673 \\
\hline 14 & S25-C5 - 45 & $152^{\prime} 152 \times 500$ & 5 & 45 & 0.0479 & 0.0427 & 0.045509 & 0.002391 \\
\hline 15 & S-NC - 35 & $152^{\prime} 152 \times 500$ & 0 & 35 & 0.0363 & 0.0367 & 0.036372 & $-7.25 \mathrm{E}-05$ \\
\hline 16 & S-NC - 40 & $152^{\prime} 152 \times 500$ & 0 & 40 & 0.0375 & 0.0387 & 0.038789 & -0.00129 \\
\hline 17 & S25-C4 - 35 & $152^{\prime} 152 \times 500$ & 4 & 35 & 0.0523 & 0.0529 & 0.055857 & -0.00356 \\
\hline 18 & S25-C4 - 40 & $152^{\prime} 152 \times 500$ & 4 & 40 & 0.0543 & 0.0552 & 0.056222 & -0.00192 \\
\hline 19 & S25-C4 - 35 & $152^{\prime} 152 \times 500$ & 4 & 35 & 0.0576 & 0.0595 & 0.055857 & 0.001743 \\
\hline 20 & S25-C4- 40 & $152^{\prime} 152 \times 500$ & 4 & 40 & 0.0583 & 0.0597 & 0.056222 & 0.002078 \\
\hline 21 & S38-C4 - 35 & $152^{\prime} 152 \times 500$ & 4 & 35 & 0.0594 & 0.0502 & 0.055857 & 0.003543 \\
\hline 22 & S38-C4- 40 & $152^{\prime} 152 \times 500$ & 4 & 40 & 0.0612 & 0.0623 & 0.056222 & 0.004978 \\
\hline 23 & S38-C5 - 35 & $152^{\prime} 152 \times 500$ & 5 & 35 & 0.0687 & 0.058 & 0.065866 & 0.002834 \\
\hline 24 & S38-C5 - 40 & $152^{\prime} 152 \times 500$ & 5 & 40 & 0.0693 & 0.062 & 0.053632 & 0.015668 \\
\hline 25 & R-NC - 35 & $203^{\prime} 152 \times 500$ & 0 & 35 & 0.0389 & 0.0401 & 0.038354 & 0.000546 \\
\hline 26 & R-NC - 40 & $203^{\prime} 152 \times 500$ & 0 & 40 & 0.042 & 0.0437 & 0.041014 & 0.000986 \\
\hline 27 & R25-C3 - 35 & $203^{\prime} 152 \times 500$ & 3 & 35 & 0.039 & 0.042 & 0.040239 & -0.00124 \\
\hline 28 & R25-C3 - 40 & $203^{\prime} 152 \times 500$ & 3 & 40 & 0.042 & 0.0706 & 0.042401 & -0.0004 \\
\hline 29 & R38-C3 - 35 & $203^{\prime} 152 \times 500$ & 3 & 35 & 0.0421 & 0.0501 & 0.040239 & 0.001861 \\
\hline 30 & R38-C3 - 40 & $203^{\prime} 152 \times 500$ & 3 & 40 & 0.0437 & 0.069 & 0.042401 & 0.001299 \\
\hline
\end{tabular}




\begin{tabular}{|c|c|c|c|c|c|c|c|c|}
\hline 31 & R5-C5 - 40 & $203^{\prime} 152 \times 500$ & 5 & 40 & 0.042 & 0.0482 & 0.043001 & -0.001 \\
\hline 32 & R5-C5 - 45 & $203^{\prime} 152 \times 500$ & 5 & 45 & 0.0443 & 0.0584 & 0.04366 & 0.00064 \\
\hline 33 & R25-C4 - 40 & $203^{\prime} 152 \times 500$ & 4 & 40 & 0.0421 & 0.043 & 0.042742 & -0.00064 \\
\hline 34 & $\mathrm{R} 25-\mathrm{C} 4-45$ & $203^{\prime} 152 \times 500$ & 4 & 45 & 0.0443 & 0.0449 & 0.043648 & 0.000652 \\
\hline 35 & S5-A3 - 40 & $152^{\prime} 152 \times 500$ & 3 & 40 & 0.0489 & 0.0501 & 0.045052 & 0.003848 \\
\hline 36 & S5-A3 - 45 & $152^{\prime} 152 \times 500$ & 3 & 45 & 0.0507 & 0.0462 & 0.046621 & 0.004079 \\
\hline 37 & S5-A6 - 44 & $152^{\prime} 152 \times 500$ & 6 & 40 & 0.0498 & 0.0412 & 0.049619 & 0.000181 \\
\hline 38 & S5-A6 - 40 & $152^{\prime} 152 \times 500$ & 6 & 45 & 0.0516 & 0.043 & 0.049941 & 0.001659 \\
\hline 39 & S5-A12 - 40 & $152^{\prime} 152 \times 500$ & 12 & 40 & 0.0522 & 0.0612 & 0.052299 & $-9.90 \mathrm{E}-05$ \\
\hline 40 & S5-A12- 45 & $152^{\prime} 152 \times 500$ & 12 & 45 & 0.0542 & 0.0714 & 0.052651 & 0.001549 \\
\hline 41 & $\mathrm{~S} 25-\mathrm{A} 3-40$ & $152^{\prime} 152 \times 500$ & 3 & 40 & 0.0482 & 0.0467 & 0.045052 & 0.003148 \\
\hline 42 & S25-A3 - 45 & $152^{\prime} 152 \times 500$ & 3 & 45 & 0.0512 & 0.0466 & 0.046621 & 0.004579 \\
\hline 43 & S25-A6 - 40 & $152^{\prime} 152 \times 500$ & 6 & 40 & 0.0501 & 0.0485 & 0.049619 & 0.000481 \\
\hline 44 & S25-A6 - 45 & $152^{\prime} 152 \times 500$ & 6 & 45 & 0.0512 & 0.0495 & 0.049941 & 0.001259 \\
\hline 45 & S25-A9- 40 & $152^{\prime} 152 \times 500$ & 9 & 40 & 0.0521 & 0.0461 & 0.051741 & 0.000359 \\
\hline 46 & S25-A9- 45 & $152^{\prime} 152 \times 500$ & 9 & 45 & 0.0533 & 0.047 & 0.052436 & 0.000864 \\
\hline 47 & S25-A12 - 40 & $152^{\prime} 152 \times 500$ & 12 & 40 & 0.0512 & 0.0451 & 0.052299 & -0.0011 \\
\hline 48 & S25-A $12-45$ & $152^{\prime} 152 \times 500$ & 12 & 45 & 0.0533 & 0.0463 & 0.052651 & 0.000649 \\
\hline 49 & S38-A6- 40 & $152^{\prime} 152 \times 500$ & 6 & 40 & 0.051 & 0.0485 & 0.049619 & 0.001381 \\
\hline 50 & S38-A6- 45 & $152^{\prime} 152 \times 500$ & 6 & 45 & 0.054 & 0.0495 & 0.049941 & 0.004059 \\
\hline 51 & S38-A9 - 40 & $152^{\prime} 152 \times 500$ & 9 & 40 & 0.0512 & 0.0452 & 0.051741 & -0.00054 \\
\hline 52 & S38-A9 - 45 & $152^{\prime} 152 \times 500$ & 9 & 45 & 0.0529 & 0.0469 & 0.052436 & 0.000464 \\
\hline 53 & S5-A9- 40 & $152^{\prime} 152 \times 500$ & 9 & 40 & 0.0511 & 0.05 & 0.051741 & -0.00064 \\
\hline 54 & S5-A9- 45 & $152^{\prime} 152 \times 500$ & 9 & 45 & 0.0538 & 0.0517 & 0.052436 & 0.001364 \\
\hline 55 & $\mathrm{C} 100-\mathrm{C} 2-40$ & $150^{\prime} 300$ & 2 & 40 & 0.0734 & 0.0744 & 0.070843 & 0.002557 \\
\hline 56 & C100-C2 - 45 & $150^{\prime} 300$ & 2 & 45 & 0.0742 & 0.0752 & 0.06949 & 0.00471 \\
\hline 57 & C150-A3 - 40 & $150^{\prime} 300$ & 3 & 40 & 0.0463 & 0.0621 & 0.052827 & -0.00653 \\
\hline 58 & C150-A3 - 45 & $150^{\prime} 300$ & 3 & 45 & 0.0473 & 0.0728 & 0.051922 & -0.00462 \\
\hline 59 & C150-A6 - 40 & $150^{\prime} 300$ & 6 & 40 & 0.0552 & 0.0682 & 0.052083 & 0.003117 \\
\hline 60 & C150-A6 - 45 & $150^{\prime} 300$ & 6 & 45 & 0.0589 & 0.0737 & 0.051756 & 0.007144 \\
\hline 61 & C150-A9 - 40 & $150 \prime 300$ & 9 & 40 & 0.0683 & 0.0671 & 0.070838 & -0.00254 \\
\hline 62 & C150-A9 - 45 & $150^{\prime} 300$ & 9 & 45 & 0.0709 & 0.0706 & 0.071766 & -0.00087 \\
\hline 63 & C150-A12 - 40 & $150^{\prime} 300$ & 12 & 40 & 0.081 & 0.07 & 0.080127 & 0.000873 \\
\hline 64 & C150-A12 - 45 & $150^{\prime} 300$ & 12 & 45 & 0.0844 & 0.0714 & 0.083661 & 0.000739 \\
\hline
\end{tabular}



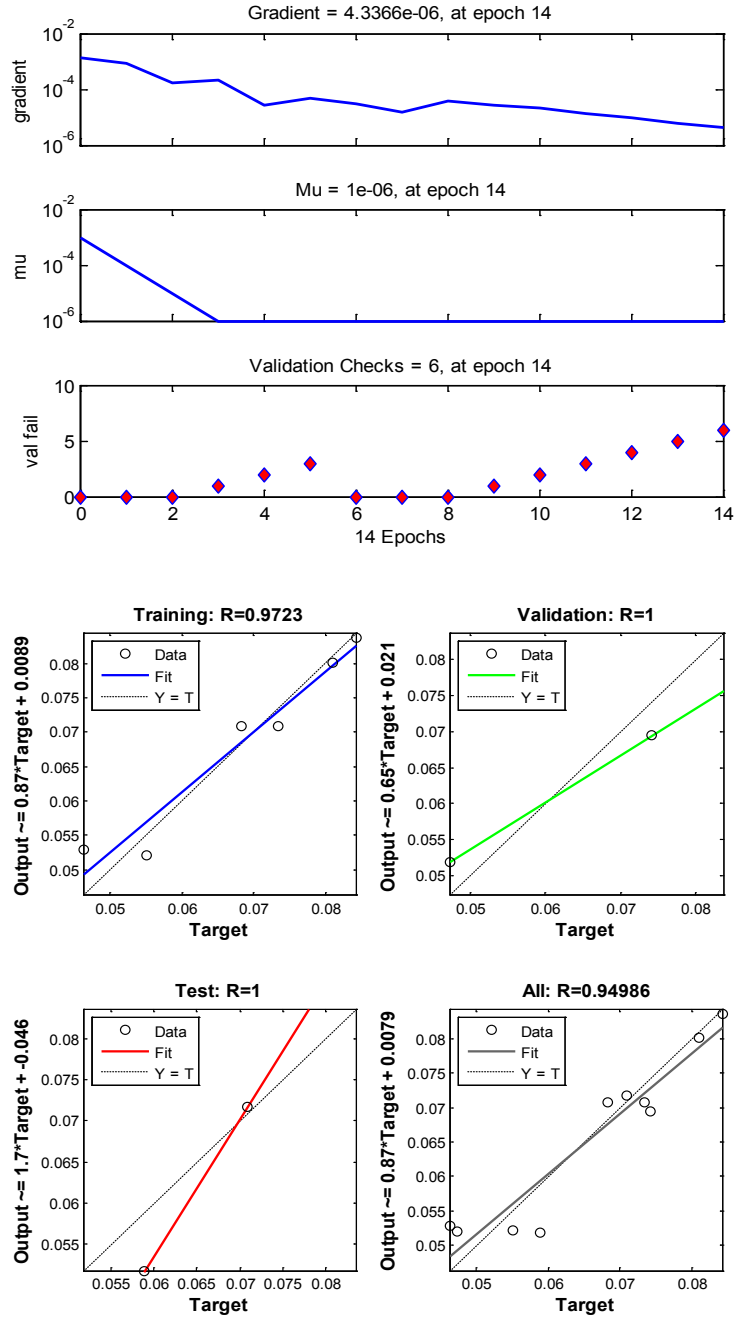

Figure 6. Regressions of training, validation and test data for circular shaped column.

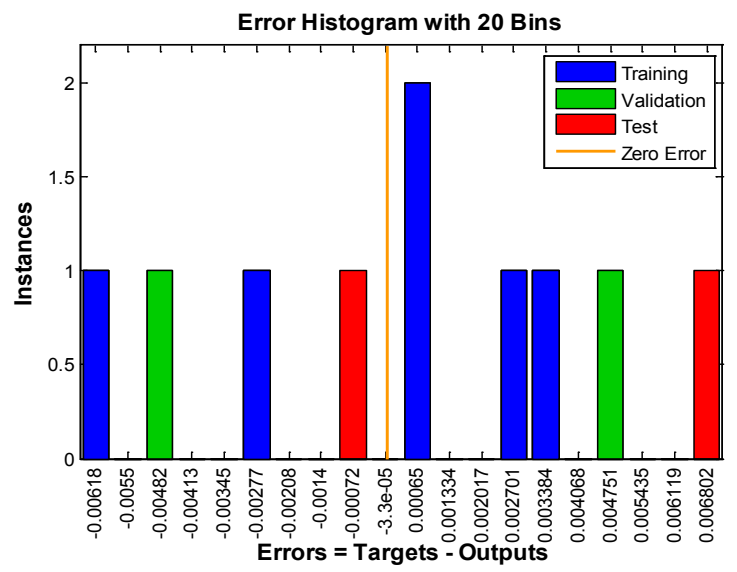

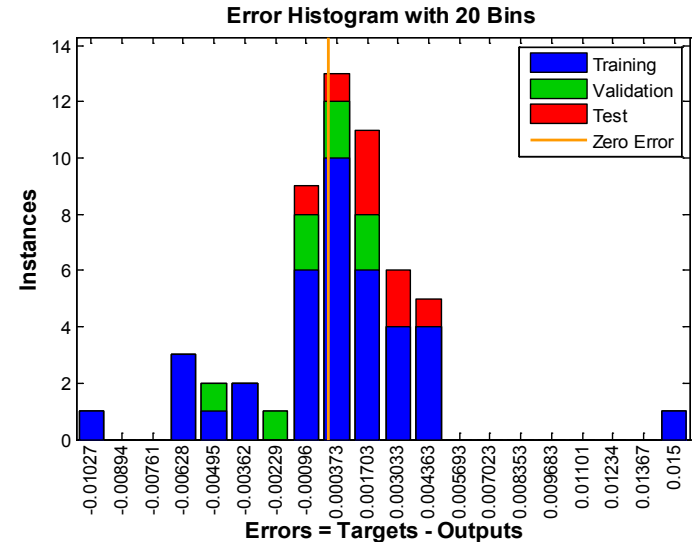

Figure 7. Error histogram data simulated by ANN for Square and rectangular and circular columns.

\section{Conclusions}

The compressive strength of FRP-confined concrete column was obtained using ANSYS and ANN for sixty four specimens. The effective neural network approach was selected in order to verify the performance of the network. The results from ANN simulations were compared with the compressive stress of specimens obtained from ANSYS output. The average error for the Artificial Neural Network model for predicting the experimental stress and analytical stresswas lower by $4 \%$. The error was very less in the order of $\pm 1 \%$ of the experimental and analytical stresses. Also values simulated by the ANN model set spread around the $45^{\circ}$ line which shows that the predicted values were neither over-estimated nor under-estimated. Thus ANN model is used to predict the compressive stress values of FRP wrapped concrete columns under compressive load.

\section{References}

1. Mirmiran A, Shahawy M. Behavior of concrete columns Confined by Fiber Composites. Journal of Structural Engineering. 1997; 123(5):583-590. https://doi.org/10.1061/ (ASCE)0733-9445(1997)123:5(583)

2. Samaan M, Mirmiran A, Shahawy M. Model of concrete confined by fiber composites. Journal of Structural Engineering. 1998; 124(9):1025-31.https://doi.org/10.1061/ (ASCE)0733-9445(1998)124:9(1025)

3. Spoelstra MR, Monti G. FRP-confined concrete model. Journal of Composites for Construction. 1999; 3(3):143-50. https://doi.org/10.1061/(ASCE)1090-0268(1999)3:3(143) 
4. Parvin A, Wang W. Behavior of FRP jacketed concrete columns under eccentric loading. Journal of Composite for Construction. 2000; 4(3):146-52. https://doi.org/10.1061/ (ASCE)1090-0268(2001)5:3(146)

5. Yang X, Nanni A, Chen G. Effect of corner radius of performance of externally bonded FRP reinforcement. Non-metallic Reinforcement for concrete StructuresFRPRCS-5; 2001. p. 197-204.

6. Cole C, Belarbi A. Coefficient characteristics of rectangular FRP- Jacketed RC Columns. Proceedings of the 5th International Symposium on Fiber-Reinforced Polymer for Reinforced Concrete Structures (FRPRCS - 5); 2001. p. 828-32.

7. Huang CS, Yeh YK, Liu GY, Hu HT, Tsai KC, Weng YT, Wang $\mathrm{SH}, \mathrm{Wu} \mathrm{MH}$. Axial load behaviour of stiffened concretefilled steel columns. Journal of Structural Engineering. 2002; 128(9):1222-230. https://doi.org/10.1061/(ASCE)07339445(2002)128:9(1222)

8. Hu HT, Huang CS, Wu MH, Wu YM. Nonlinear analysis of axially loaded concrete- filled tube columns with confinement effect. Journal of structural Engineering. 2003; 129(10):1322-9. https://doi.org/10.1061/(ASCE)07339445(2003)129:10(1322)
9. Rochettee P, Labossiere P. Axial testing of Rectangular column models confined with composites. Journal of Composites for Construction. 2000; 4(3):129-36. https://doi.org/10.1061/(ASCE)1090-0268(2000)4:3(129)

10. Sangeetha P. Analysis of FRP wrapped concrete columns under uniaxial compression. Journal of Scientific and Industrial Research. 2007; 66:235-242.

11. Sangeetha P, Sumathi R. Behaviour of glass fiber wrapped concrete columns under uniaxial compression. International Journal of Advanced Engineering Technology. 2010; 1(1):74-83.

12. Sangeetha P, Senthil R. Experimental behaviour of steel tubular columns for varying in filled concrete. Archives of Civil Engineering. 2017; 63(4):149-60. https://doi. org/10.1515/ace-2017-0046a

13. Sangeetha $P$, Ashwin Muthuraman RM, Dachina G, Dhivya M, Janani S, Madumathi S. Behaviour of concrete filled steel tubes. Journal of Informatics and Mathematical Sciences. 2018; 10(1-2):297-304. https://doi.org/10.26713/jims. v10i1-2.1056 